\title{
QUANTITATIVE ASSESSMENT OF BONE REMODELLING AND OSTEOPHYTOGENESIS IN MURINE OSTEOARTHRITIS
}

\author{
Patricia Borges $^{1}$, Tonia Vincent ${ }^{2}$ and Massimo Marenzana ${ }^{1,2}$ \\ 'Department of Bioengineering, Imperial College London, UK; ${ }^{2}$ The Kennedy Institute of Rheumatology, \\ University of Oxford, UK. \\ | p.das-neves-borges11@imperial.ac.uk | m.marenzana@imperial.ac.uk |
}

\section{BACKGROUND}

Subchondral bone $(\mathrm{Sb})$ remodelling and osteophyte growth are widely recognised hallmarks of knee osteoarthritis $(O A)$, although their contribution to the disease is not fully understood. Murine models, for their amenability to genetic studies, have been instrumental to identify key pathways modulating $\mathrm{OA}$ and are becoming essential tools to discover new therapeutic targets. The interaction between bone and cartilage is not understood, with genetically chondroprotected mice further complicating this relationship as they still show prominent osteophytogenesis irrespective of cartilage damage.

AIM

To use 3D automated image analysis methods to characterize quantitatively $\mathrm{Sb}$ remodelling and osteophytogenesis during the progression of murine $\mathrm{OA}$

\section{D AUTOMATED MICROSTRUCTURAL ANALYSIS}

Sb.PI and Tb compartmentalization is based on a threshold criterion (cortical bone volume fraction $>90 \%$ ):

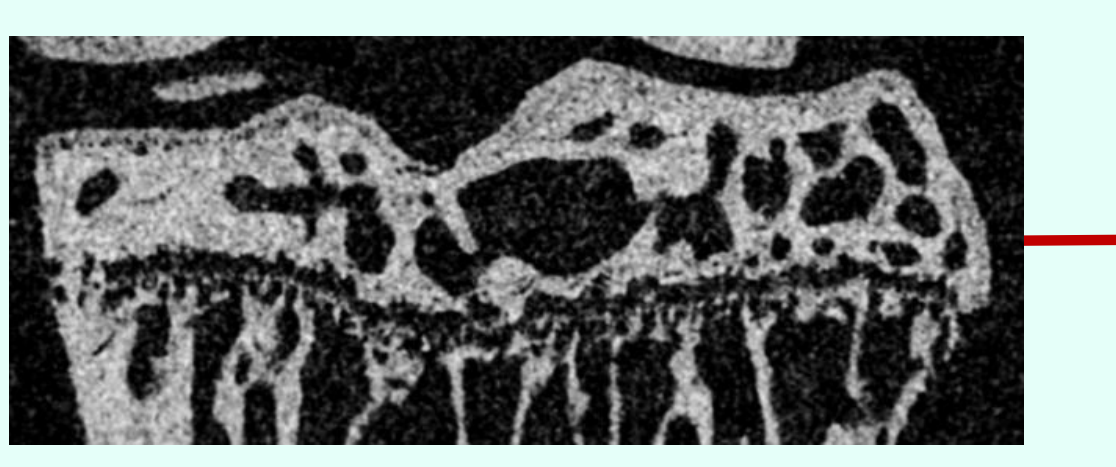

\section{(1) Unprocessed} (1) Unprocessed $\mu \mathrm{C}$ image of mouse tibia (middle coronal plane)

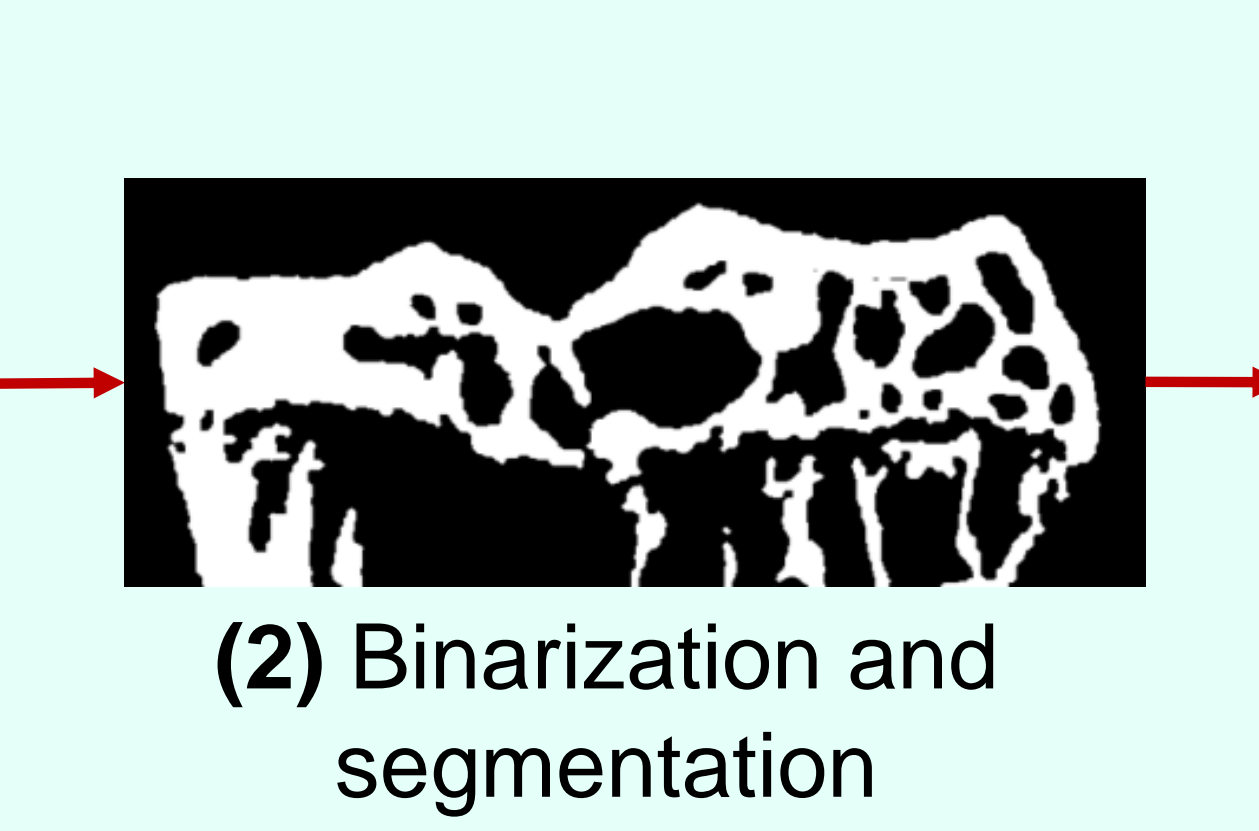

Cortical Compartment
Trabecular Compartment

(4) Volumes of

interest enclosed
in the masks

\begin{tabular}{lrrr}
\hline MTP - Sb.PI. & MTP - Tb & LTP - Sb.PI. & LTP - Tb \\
& & & \\
& & & \\
\hline
\end{tabular}

\section{RESULTS}

TEMPORAL CHANGES IN SUBCHONDRAL BONE STRUCTURE

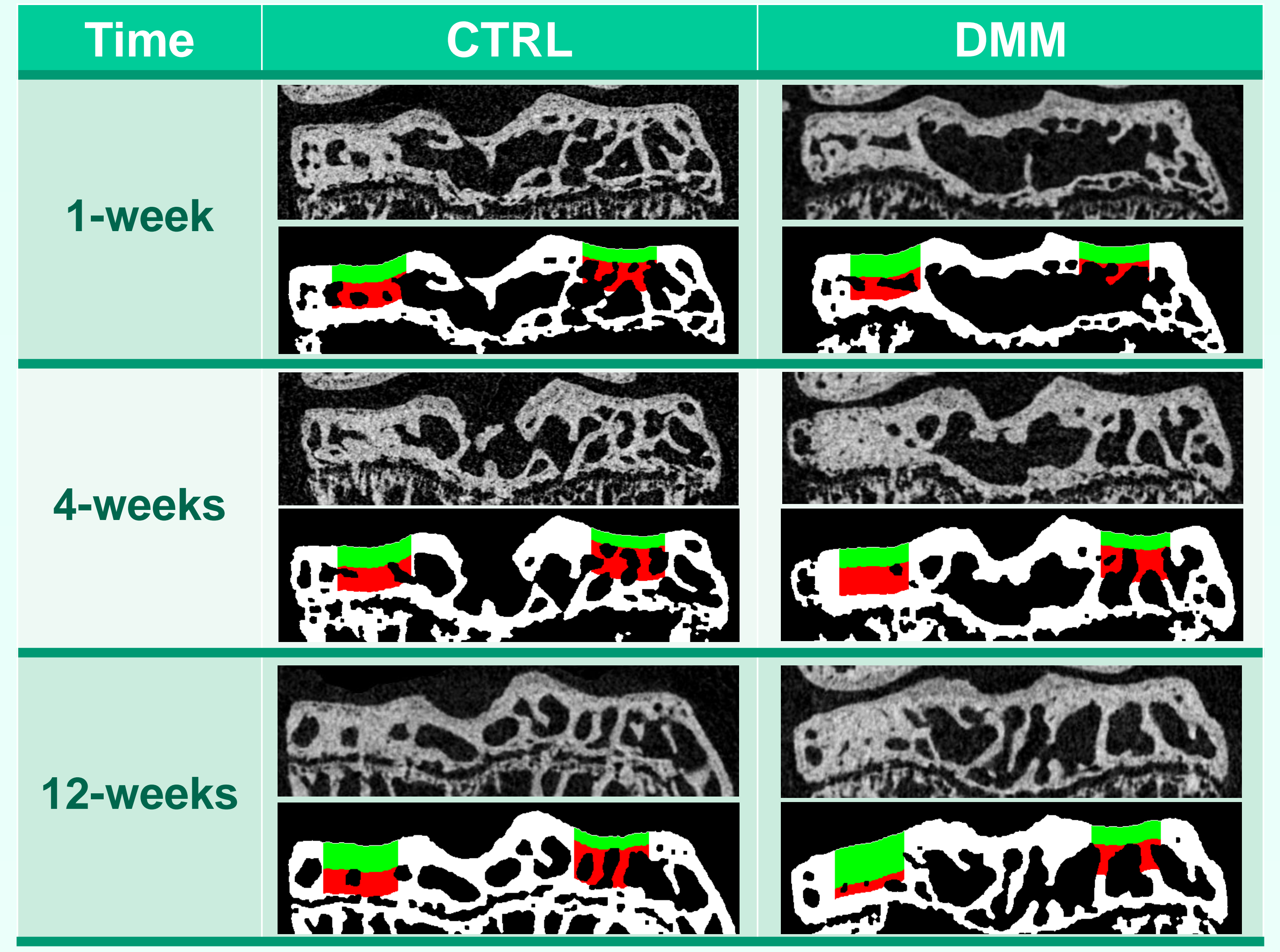

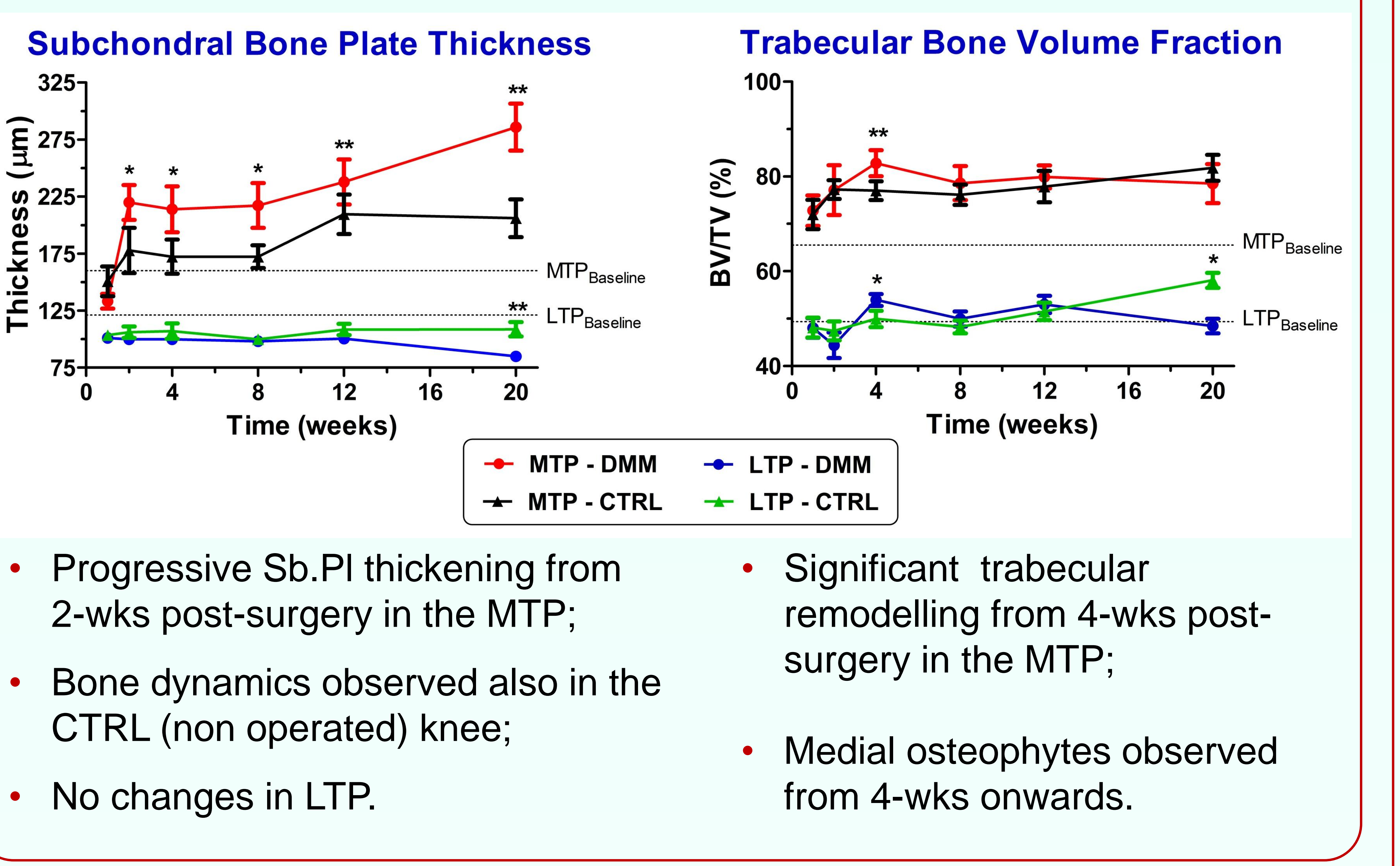

\section{CONCLUSIONS}

- High-resolution $\mu \mathrm{CT}$ can been used to assess quantitatively bone microarchitecture in the tibial epiphysis;

- Automated (hence quantitative) image analysis identified Sb.P . thickening, Tb sclerosis and osteophyte growth, from early stages in the DMM mouse model of OA:

- The proposed method significantly increases throughput and sensitivity in the assessment of subchondral bone remodelling in murine OA.

\section{METHODS}

Murine OA model. Six groups of C57BL/6J mice (10-week old, $n=6$ ) underwent surgical destabilisation of the medial meniscus (DMM) in right knee joints and were euthanized 1, 2, 4, 8, 12 and 20-weeks post-operatively. Imaging. Dissected tibiae were imaged using $\mu \mathrm{CT}$ (Skyscan 1172, $5 \mu \mathrm{m} / \mathrm{pixel}$ ) and reconstructions were analysed by an automated software (Matlab), which maps subchondral bone plate (Sb.PI.) and trabecular bone (Tb) compartments in the medial (MTP) and lateral tibial plateau (LTP) load-bearings and computes thickness (Sb.PI.Th) and volume fraction (Tb BV/TV). Whole tibial epiphysis and osteophytes were segmented and their volume was computed. A group of naïve C57BL/6J animals $(n=6)$ was used to set a healthy baseline for all the measurements. Results were evaluated comparing operated side $(\mathrm{DMM})$ vs. contralateral $(\mathrm{CTRL})$ over time ( ${ }^{*} \mathrm{p} \leq 0.05$, DMM vs. CTRL).

Novel ex-vivo $\mu \mathrm{CT}$ imaging method for 3D assessment of subchondral bone remodelling in the mouse model of OA

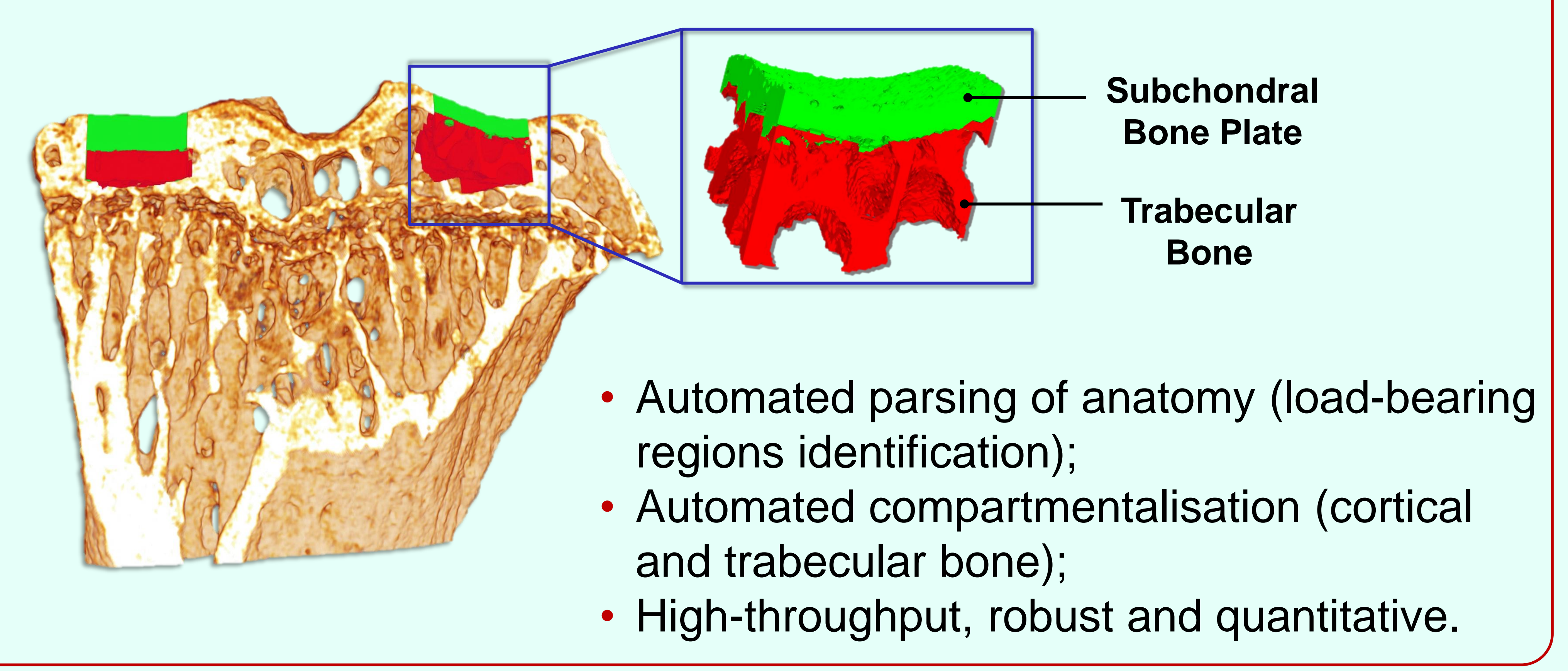

\section{OSTEOPHYTOGENESIS ASSESSMENT}

Osteophytogenesis is characterized by new bone formation in the margins of the joint leading to an overall epiphyseal expansion. In the DMM model, osteophytes can be clearly identified on the medial side of the tibia.
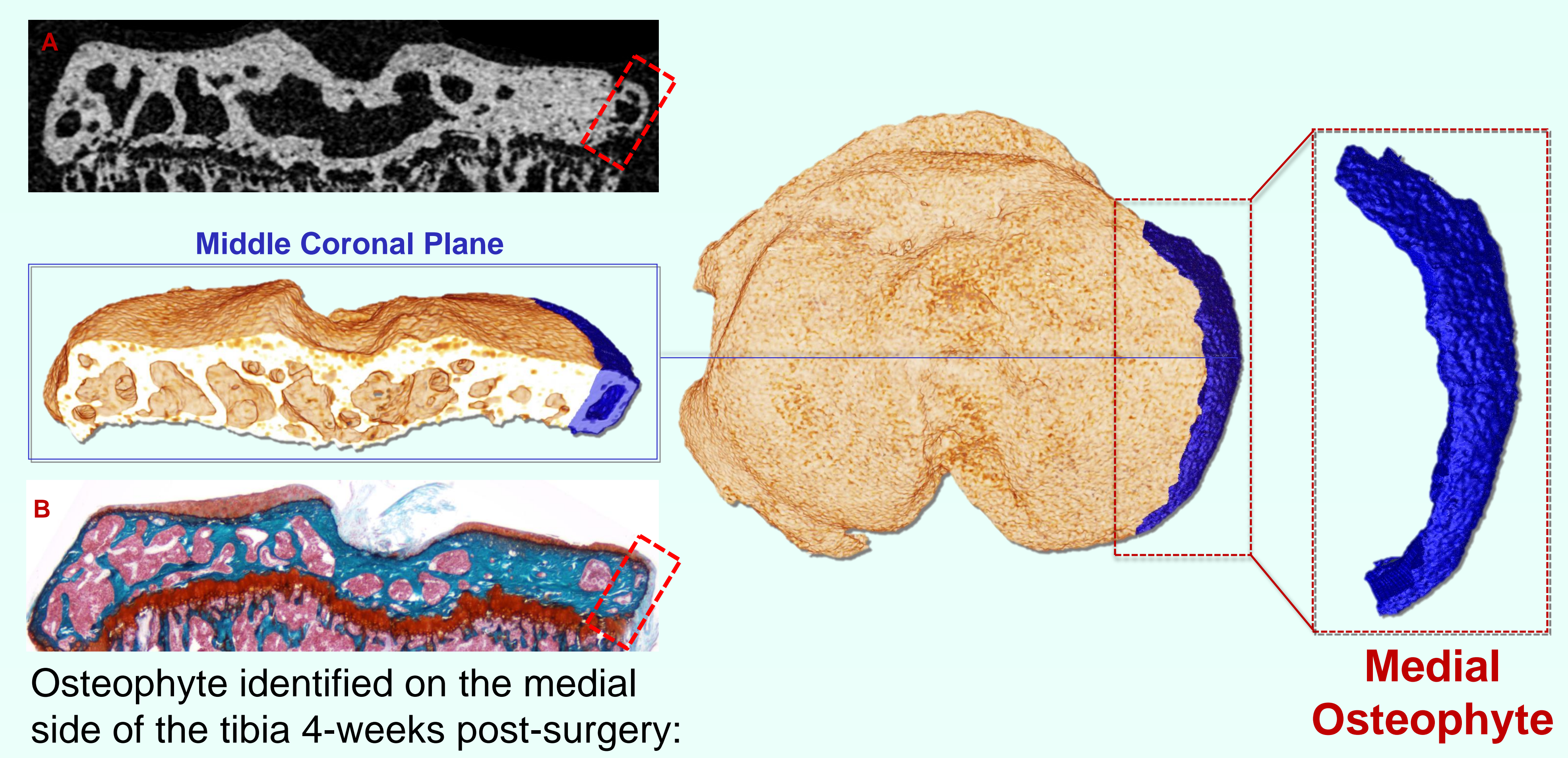

A) $\mu \mathrm{CT}$ and B) histological section.

Whole Epiphysis Volume

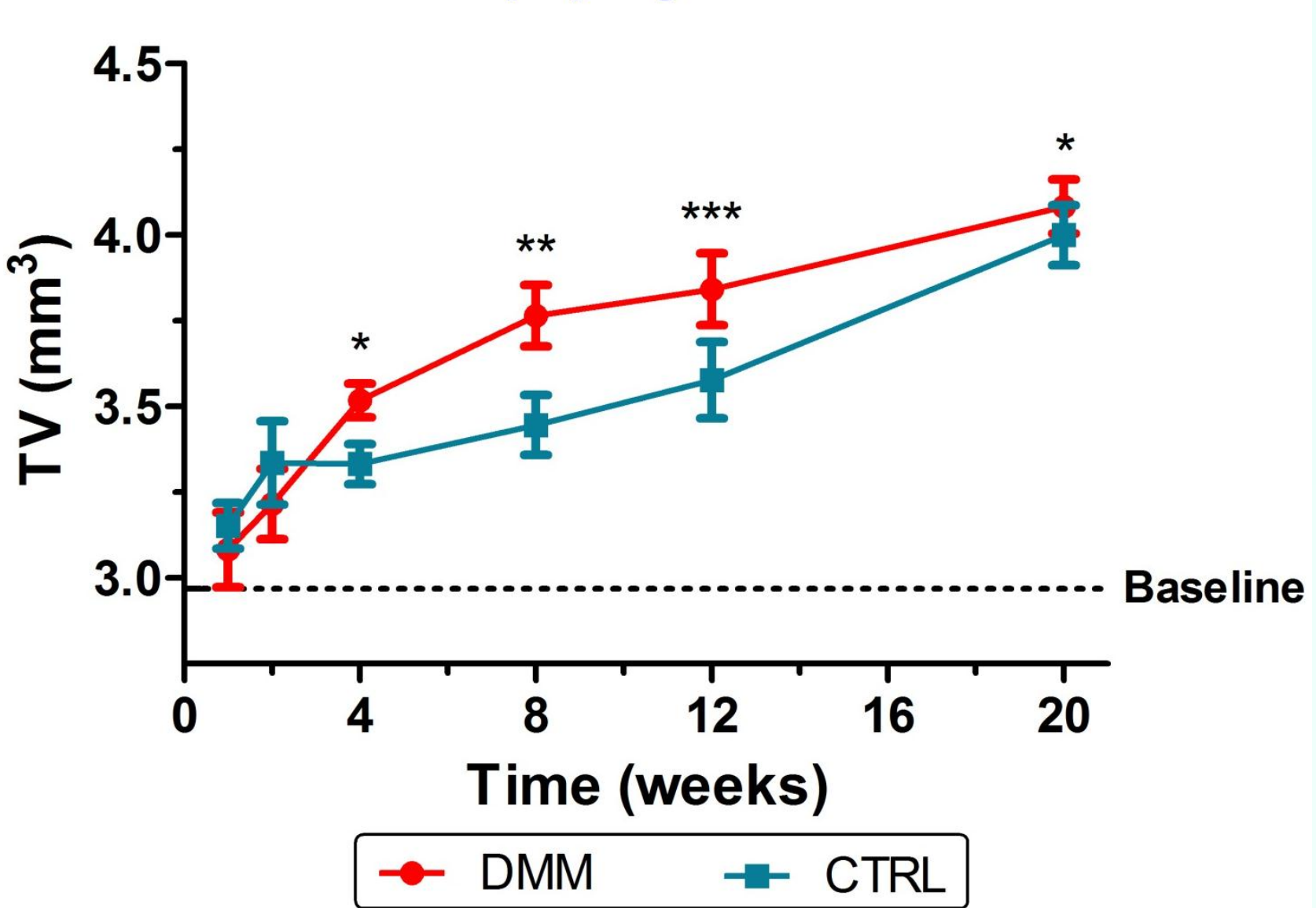

Medial Osteophyte Volume

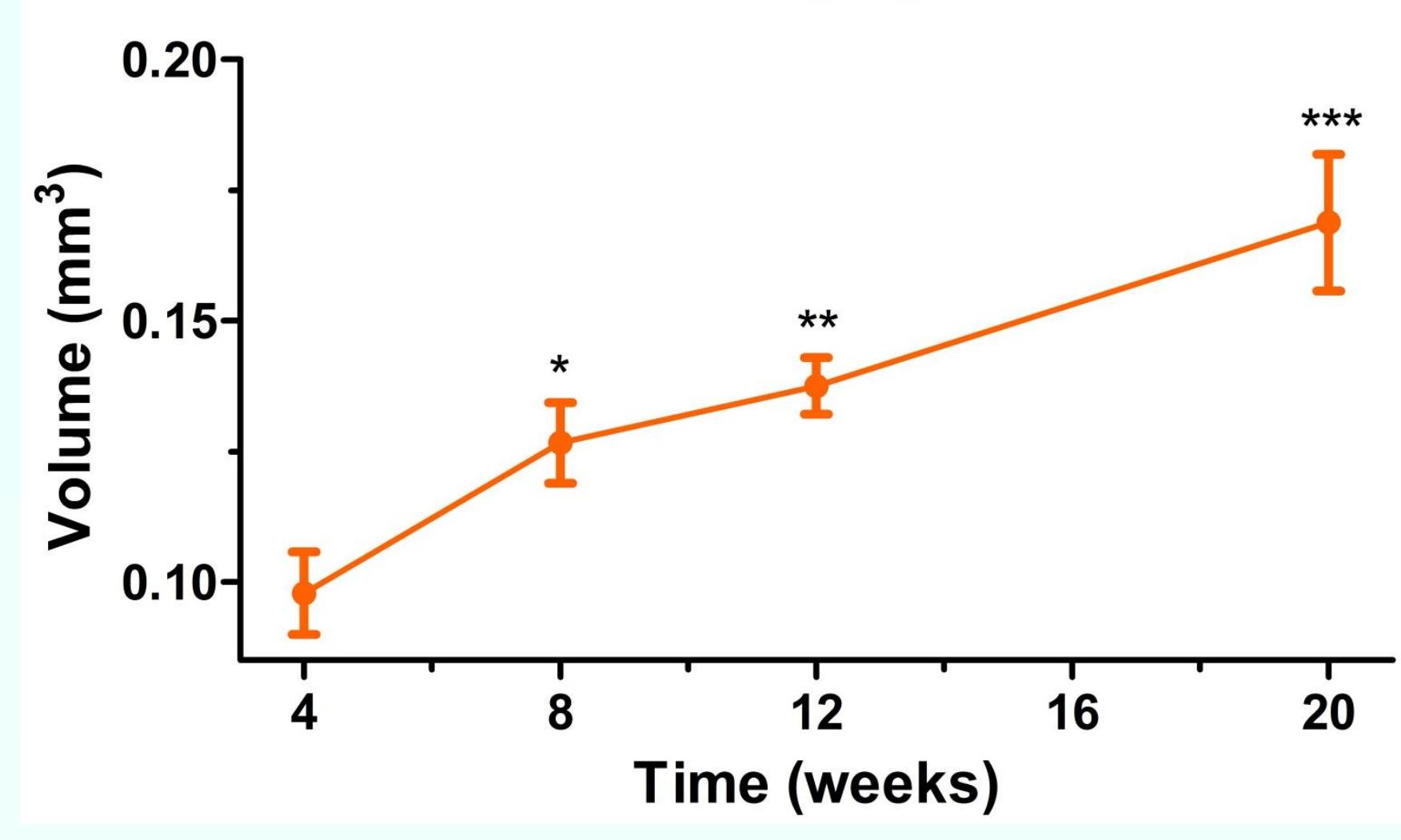

Osteophyte volume increases over time (mineralised phase).

detected by measuring the volume

(TV) of the whole epiphysis.

Increased from 4-wks.

Osteophyte volume contributes $\sim 3 \%$ for whole epiphyseal volume at 4-wks and up to $4 \%$ at 20 -wks post-surgery;

- Additional shape modelling might contributed to the remaining epiphyseal expansion $(6 \%$ increased at 4 -wks in DMM compared to CTRL).

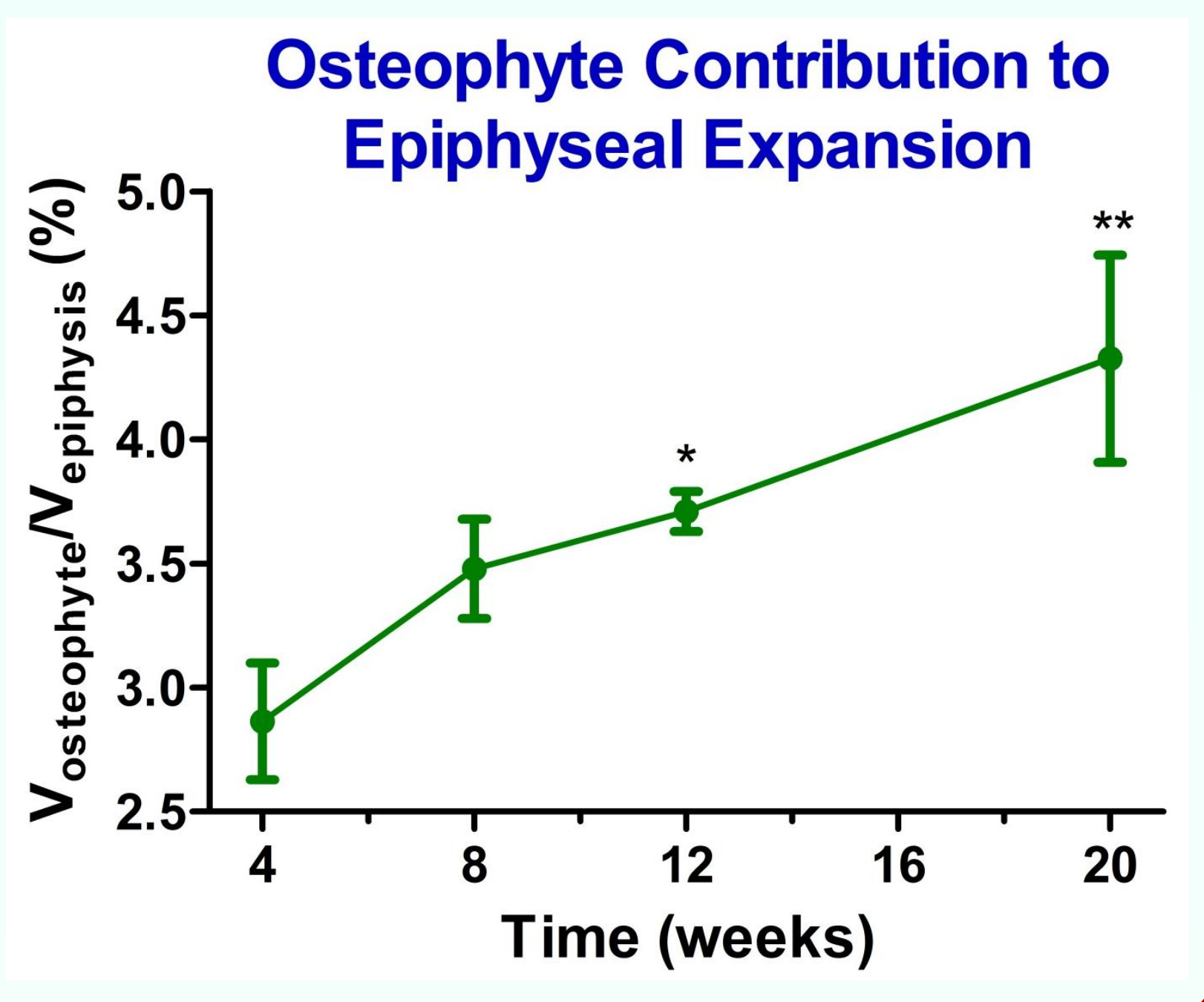

Conflict of Interest: The authors have no conflicts of interest to disclose.

ACKNOWLEDGEMENTS

The authors would like to thank Welcome Trust /EPSRC (Medical Engineering Solutions in Osteoarthritis Centre of Excellence) and the Kennedy Institute of Rheumatology Trust for funding this research. 